\title{
Víctima y verdugo: características de personalidad y psicopatología de los receptores y perpetradores de bullying
}

\author{
Paula Etkin \\ etkin@uji.es \\ Jordi Ortet Walker \\ jortet@uji.es \\ Verónica Vidal Arenas \\ vvidal@uji.es \\ Generós Ortet \\ ortet@uji.es \\ Laura Mezquita \\ Imezquit@uji.es
}

\section{Resumen}

Antecedentes/Objetivo: El acoso escolar o bullying es una conducta en la que se ven envueltos la mayoría de los jóvenes, como perpetrador, víctima o ambos, y que se ha asociado tanto a rasgos de personalidad como a la psicopatología. El objetivo de este trabajo fue estudiar en una muestra de adolescentes la asociación entre las dimensiones de personalidad del modelo de cinco factores y los síntomas psicopatológicos más comunes, con los roles de víctimas y perpetradores de acoso escolar.

Método: Participaron 231 adolescentes de 13-18 años (media de edad $=15,25 ; \quad D T=1,00 ; 52 \%$ chicas). Completaron cuestionarios sobre características sociodemográficas, psicopatología, personalidad y una escala de conductas de acoso escolar. Se realizaron análisis de correlación y regresión para explorar las asociaciones de la personalidad y los síntomas internalizantes y externalizantes con las conductas de acoso y situaciones de víctima de acoso.

Resultados: Las regresiones mostraron que realizar más conductas de acoso se asociaba con ser varón, poco amable, presentar psicopatología externalizante $\mathrm{y}$, en menor medida, ser poco abierto a la experiencia y puntuar alto en neuroticismo. Por otra parte, mayores puntuaciones en la escala de victimización se asociaron con ser varón, poco amable y presentar elevadas puntuaciones en psicopatología externalizante e internalizante.

Discusión: Estos hallazgos replican los resultados de estudios previos, mostrando la relativa similitud en los perfiles psicopatológicos de los adolescentes, víctimas y perpetradores, y van encaminados a esclarecer las 
relaciones entre los constructos estudiados, lo que puede contribuir a desarrollar programas de prevención y tratamiento del acoso escolar.

Palabras clave: acoso escolar, adolescentes, personalidad, psicopatología.

\begin{abstract}
Background/Objective: Bullying is a behavior in which many young people are involved, as a perpetrator, victim or both, and that has been associated with personality traits and psychopathology. The aim of this work was to examine the association between the Five-Factor Model personality dimensions, and the most common psychopathological symptoms, with the roles of victims and perpetrators of bullying in a sample of adolescents.

Method: A total of 231 adolescents aged 13-18 years (mean age=15.25, $S D=1.00,52 \%$ girls) participated in this study. They completed questionnaires on sociodemographic characteristics, psychopathology, personality and a bullying scale. Correlations and regression analyses were conducted to explore the associations of personality traits and internalizing and externalizing symptoms with harassment behaviors and victim situations of bullying.

Results: The results of the regressions showed that being male, antagonistic, presenting externalizing psychopathology and, to a lesser extent, being low in openness, and scoring high in neuroticism, were associated with performing more harassing behaviors. On the other hand, being male, antagonist, and presenting high scores in externalizing and internalizing psychopathology were associated with higher scores on the victimization scale.

Discussion: These findings are in line with previous studies, showing the relative similarity in the psychopathological profiles of adolescent victims and perpetrators of bullying, and clarify the relationships between the studied constructs, which may contribute to develop prevention and treatment in bullying programs.
\end{abstract}

Keywords: bullying, adolescents, personality, psychopathology.

\title{
Introducción
}

El acoso escolar o bullying es una conducta en la que se ven envueltos muchos jóvenes $(70,9 \%)$, como perpetrador, como víctima o en ambos roles (Goldbach, Sterzing y Stuart 2018). Los estudios en este campo han encontrado que son los varones quienes utilizan mayormente este tipo de violencia y que la victimización y las pobres relaciones sociales serían algunos factores predictores de problemas emocionales en los adolescentes (Ramírez, Ramírez-Pérez, Arellano y Trejo Vázquez 2016). Respecto a los diferentes roles implicados, algunos niños y niñas que realizan conductas de acoso son, a su vez, víctimas del acoso de otros y se ha visto que estos grupos comparten ciertos procesos psicológicos y características que subyacen a sus comportamientos (van Dijk, Poorthuis y Malti 2017).

La personalidad se ha relacionado con los comportamientos de acoso, fundamentalmente desde el modelo de los cinco factores (McCrae y Costa 2010). Un metaanálisis (Mitsopoulou y Giovazolias 2015) sugiere que, cuando se comparan las características de personalidad entre víctimas y agresores, ambos perfiles se 
caracterizan por bajos niveles de amabilidad y de responsabilidad, así como por un alto neuroticismo o inestabilidad emocional. En cuanto a las diferencias, el perfil de acosador o bully, estaría asociado a una baja empatía (Mitsopoulou y Giovazolias 2015), impulsividad, baja amabilidad (Volk, Schiralli, Xia, Zhao y Dane 2018) y conductas maquiavélicas (van Geel, Goemans, Toprak y Vedder 2017). Por otra parte, hay estudios que señalan que tanto las víctimas como las vínctimas-agresores presentan un mayor neuroticismo y que, además, las víctimas presentan mayor apertura a la experiencia y amabilidad; mientras que los adolescentes que no participan en ninguno de estos dos roles puntúan más alto en responsabilidad y amabilidad (Alonso y Romero 2017). Además, en estudios con adultos, se indicó que los rasgos de personalidad pueden funcionar tanto como predictores, preventores y víctimas por bullying en el trabajo (Nielsen y Knardahl 2015; Plopa, Plopa y Skuzińska 2017).

En cuanto a la relación entre acoso escolar y síntomas psicopatológicos, estudios previos sugieren que los sujetos implicados presentan riesgos a diferentes problemas a lo largo de la vida. Así, estudiantes involucrados en estas conductas, en cualquiera de los roles, presentan más síntomas y trastornos en general que los estudiantes no involucrados (Kelly et al. 2015). Fundamentalmente, las víctimas se encontrarían frente a un mayor riesgo de presentar trastornos y síntomas internalizantes como ansiedad y depresión (Bang y Park 2017; Klomek, Sourander y Elonheimo 2015; Silberg et al. 2016). Los perpetradores de acoso escolar padecerían mayores problemas externalizantes, manifestando síntomas como conductas antisociales, agresividad y consumo de sustancias, en comparación con las víctimas de acoso escolar y los estudiantes no implicados en ellas (Kelly et al. 2015; Sterzing et al. 2017). Por otra parte, los problemas internalizantes, como ansiedad, síntomas depresivos o postraumáticos, se asociaron más a un perfil dual de víctima-perpetrador de bullying, sobre todo al combinarse con algunos problemas externalizantes (Farmer et al. 2015; Kelly et al. 2015; Sterzing et al. 2017). Otro estudio (Silberg et al. 2016) encuentra que ser víctima de acoso escolar en la niñez representaría un trauma ambiental significativo y debería ser tenido en cuenta al evaluar la salud mental tanto de niños como de jóvenes. El bullying también se presenta como un factor de riesgo independiente para el desarrollo de futuras conductas externalizantes (Hwang, Kim, Koh, Bishop y Leventhal 2017). Involucrarse en conductas de acoso, en cualquiera de los roles implicados, sería un evento vital estresante y se podría constituir en un catalizador de dificultades psicosociales (Swearer y Hymel 2015).

En general la mayor frecuencia de exposición a conductas de acoso escolar se ha asociado a más problemas de salud en la edad adulta (Klomek, Sourander y Elonheimo 2015). Asimismo, diferentes estudios sugieren que el acoso escolar se presenta en diferentes grupos, pero que la sintomatología expresada por la víctima varía según la edad y que existe un mayor riesgo de sufrir acoso entre niños de contextos socioeconómicos desfavorecidos (Tippett y Wolke 2015). Por otra parte, estudios con modelos de regresión logística multivariante revelaron que la victimización por bullying se asoció significativamente con los intentos de suicidio, incluso después de ajustar las características demográficas, aportando más evidencia a la idea de que la victimización por bullying es un factor de riesgo para la depresión, la psicosis y los intentos de suicidio (Bang y Park 2017). De esta manera, podemos entender el acoso escolar como un fenómeno complejo que es influenciado por múltiples factores, dentro de un marco socioecológico, en el que interactúan dinámicamente pares, familia y comunidad (Swearer y Hymel 2015).

$\mathrm{Ha}$ sido sugerido que, al estudiar las asociaciones entre bullying y síntomas internalizantes y externalizantes, futuros estudios deberían incluir otros factores a modo de control, para poder dar cuenta de los mecanismos detrás de estas 
asociaciones (Klomek, Sourander y Elonheimo 2015). A partir de lo expuesto, se considera que el estudio de las asociaciones entre las conductas de acoso y otras variables, como los rasgos de personalidad y distintos síntomas psicopatológicos, continúa siendo un campo relevante y en crecimiento, que requiere aportes que clarifiquen cómo las distintas variables implicadas se asocian al fenómeno. Hasta donde llega nuestro conocimiento, en base a la literatura revisada, no hay estudios en población adolescente que hayan estudiado las asociaciones entre los perfiles implicados en el bullying y un amplio abanico de síntomas psicopatológicos, controlando por los rasgos de personalidad.

El objetivo del presente trabajo fue examinar la asociación entre los rasgos de personalidad y los síntomas psicopatológicos más comunes en una muestra de adolescentes, así como también entre los suprafactores internalizante y externalizante, con los roles de víctimas y perpetradores de acoso escolar. Las asociaciones más esperables son entre el rol de acosador escolar y las conductas externalizantes, fundamentalmente agresividad y conducta antisocial; $y$, por otra parte, entre víctima de acoso y problemas internalizantes, como ansiedad y depresión. En cuanto a la personalidad, se espera hallar alto neuroticismo y baja amabilidad en ambos perfiles, con diferencias en la fuerza de las asociaciones (mayor neuroticismo en las víctimas de acoso).

\section{Método}

\section{Participantes}

En este estudio participaron 231 adolescentes de entre 13 y 18 años (media de edad=15,25 años; $D T=1,00 ; 52 \%$ chicas). Todos los participantes eran estudiantes de secundaria de un instituto público de Castellón de la Plana y la mayoría $(83,21 \%)$ eran de nacionalidad española.

\section{Instrumentos}

En primer lugar, completaron un cuestionario sobre características sociodemográficas (edad, género, nacionalidad). Luego se utilizó el Sistema de Evaluación de Niños y Adolescentes (SENA, Fernández-Pinto, Santamaría, Sánchez-Sánchez, Carrasco y del Barrio 2016), que mide distintas escalas de sintomatología psicopatológica en edades de 3 a 18 años. Para este estudio se utilizó solamente la versión de autoevaluación para adolescentes y se administraron las escalas correspondientes a sintomatología internalizante (depresión, ansiedad, ansiedad social, síntomas postraumáticos y quejas somáticas) y externalizante (conducta antisocial, conducta desafiante, problemas de control de la ira y agresividad). El formato de respuesta es una escala Likert de 0-4, que evalúa la frecuencia de los síntomas de nunca o casi nunca a siempre o casi siempre. Además, se utilizó el JS NEO-S (Ortet et al. 2010) para evaluar los cinco factores de la personalidad, siguiendo el modelo de los cinco factores, que también se contesta con una escala Likert de 0-4. Finalmente, para evaluar conductas de acoso escolar se utilizaron, por un lado, 7 ítems de la escala de problemas con los compañeros del SENA, que evalúan situaciones de víctima de acoso escolar (recibir agresión física y psicológica) y, además, se administró una escala de 7 ítems que evalúa conductas de acoso escolar como perpetrador (agresión física y psicológica), construida por los autores del estudio. Ambos instrumentos cuentan con la misma escala Likert que las otras escalas del SENA. 


\section{Procedimiento}

El equipo de investigación contactó personalmente con el instituto más cercano a la universidad, informando a sus autoridades acerca de las características y objetivos del estudio. Los participantes completaron los cuestionarios voluntariamente, con el debido consentimiento de sus padres y de la escuela, además de la aprobación del Comité Deontológico de la Universitat Jaume I (Resolución: 27-07-2015). Respondieron presencialmente en formato papel y lápiz, durante la hora de tutorías y con la posibilidad de hacer preguntas a los investigadores a cargo durante la evaluación. La confidencialidad y el anonimato de los datos fue asegurada a los participantes mediante la asignación de un código alfanumérico a los cuestionarios, la encriptación del fichero correspondiente a los datos personales y el guardado bajo llave de los cuestionarios completos.

Análisis

Se realizaron correlaciones bivariadas y análisis de regresión lineal, utilizando el programa SPSS versión 24, para explorar las asociaciones de las conductas de acoso y las situaciones de víctima de acoso con las escalas de síntomas del SENA y las dimensiones de personalidad. Los análisis de regresión se realizaron por pasos, controlando por edad y género.

\section{Resultados}

Como puede observarse en la tabla 1, las correlaciones entre los roles de acosador y víctima de acoso escolar mostraron una alta asociación, así como también entre ambos perfiles y casi todos los síntomas psicopatológicos evaluados en este estudio. Para el rol de víctima de acoso escolar, los síntomas que más se asociaron fueron depresión y agresividad; y para acosador, agresividad y conducta antisocial.

Tabla 1

Correlaciones entre roles de acoso escolar y síntomas psicopatológicos

\begin{tabular}{lll}
\hline & Acosador escolar & Víctima de acoso escolar \\
\hline Acosador escolar & - & $0,46^{* * *}$ \\
Depresión & $0,26^{* *}$ & $0,43^{* * *}$ \\
Ansiedad & $0,17^{*}$ & $0,37^{* * *}$ \\
Quejas somáticas & $0,18^{*}$ & $0,30^{* * *}$ \\
Síntomas postraumáticos & $0,29^{\star * *}$ & $0,39^{* * *}$ \\
Ansiedad social & 0,09 & $0,25^{* *}$ \\
Conducta antisocial & $0,53^{* * *}$ & $0,36^{* * *}$ \\
Problemas de control de la ira & $0,28^{* * *}$ & $0,22^{* *}$ \\
Conducta desafiante & $0,41^{* * *}$ & $0,32^{* * *}$ \\
Agresividad & $0,68^{* * *}$ & $0,42^{* * *}$ \\
Internalizante & $0,25^{* *}$ & $0,45^{* * *}$ \\
Externalizante & $0,59^{* * *}$ & $0,41^{* * *}$ \\
\hline
\end{tabular}

Nota. ${ }^{*} p<0,05,{ }^{* *} p<0,01,{ }^{* * *} p<0,001$ 
En relación con las regresiones (véase la tabla 2), puede observarse que ser chica se asoció negativa y significativamente a ambos roles de acoso escolar, pero no hubo asociaciones significativas con la edad. En relación con la personalidad, el rol de acosador se asoció a baja amabilidad, alto neuroticismo y baja apertura, mientras que el perfil de víctima se asoció de manera significativa solamente a baja amabilidad. En cuanto a los factores internalizante y externalizante de la psicopatología, el primero se asoció solo a víctima y el segundo a ambos perfiles, mayormente a acosador.

Tabla 2

Análisis de regresión entre los roles de acoso escolar y síntomas internalizantes y externalizantes

\begin{tabular}{ccccc}
\hline & \multicolumn{2}{c}{$\begin{array}{c}\text { Acosador } \\
\text { escolar }\end{array}$} & \multicolumn{2}{c}{$\begin{array}{c}\text { Víctima de acoso } \\
\text { escolar }\end{array}$} \\
\cline { 2 - 5 } & $\Delta R^{2}$ & 0 & $\Delta R^{2}$ & 0 \\
\hline Paso 1 & 0,03 & & 0,04 & \\
\hline Edad & & $-0,04$ & & 0,11 \\
Género & & $-0,16^{*}$ & & $-0,17^{*}$ \\
\hline Paso 2 & 0,23 & & 0,11 \\
\hline Neuroticismo & & $0,15^{*}$ & & 0,11 \\
Extraversión & & $-0,06$ & & $-0,10$ \\
Apertura & & $-0,14^{*}$ & & $-0,10$ \\
Amabilidad & & $-0,38^{* * *}$ & & $-0,23^{* *}$ \\
Responsabilidad & & $-0,03$ & & $-0,03$ \\
\hline Paso 3 & 0,14 & & 0,13 & \\
\hline Internalizante & & 0,09 & & $0,21^{*}$ \\
Externalizante & & $0,40^{* * *}$ & & $0,34^{* * *}$
\end{tabular}

Nota. ${ }^{*} p<0,05,{ }^{* *} p<0,01,{ }^{* * *} p<0,001$

\section{Discusión}

El objetivo de este trabajo consistió en examinar la asociación entre los rasgos de personalidad según el modelo de los cinco factores (McCrae y Costa 2010) y diferentes síntomas psicopatológicos con los roles de víctimas y perpetradores de acoso escolar en una muestra de adolescentes. De forma similar a los estudios previos, características de personalidad como baja amabilidad (Mitsopoulou y Giovazolias 2015; Sekol y Farrington 2016) y psicopatología externalizante (Eastman et al. 2018) se asociaron tanto con la conducta de acosar como con ser víctima de acoso, denotando cierta similitud entre estos perfiles. En cambio, la psicopatología internalizante se encontró más comúnmente en la víctima de acoso que en el perpetrador de bullying (Kelly et al. 2015). A diferencia de los estudios previos, aunque el neuroticismo se asoció con las conductas de acoso, no se relacionó con la victimización, sugiriendo que la asociación de la psicopatología internalizante con los síntomas de victimización no se debería a los elevados niveles de neuroticismo de las víctimas y podría deberse a otros factores y procesos, incluyendo la misma situación de victimización, en consonancia con estudios que han mostrado que los adolescentes involucrados en el acoso escolar están más expuestos a presentar síntomas 
depresivos (Díaz-Atienza, Prados Cuesta y Ruiz-Veguilla 2004; Sandoval Ato, Vilela Estrada, Mejía y Caballero Alvarado 2018).

Las bajas puntuaciones en apertura a la experiencia (i.e., personas conservadoras, poco abiertas a otras costumbres y culturas) emergen, además, como característica de personalidad a tener en cuenta en los adolescentes acosadores. Respecto a este rasgo, puede considerarse que personas poco tolerantes serían más propensas a participar en situaciones de hostigamiento a sus pares.

En relación con el sexo, de manera similar a estudios previos (Ramirez 2015), se halló que el bullying es un fenómeno que se da mayormente entre los chicos y en el que las chicas se ven menos implicadas. Dicho estudio sugirió que los chicos expresan una escasa percepción de gravedad de estas conductas, lo que colaboraría a su normalización.

En cuanto a síntomas psicopatológicos específicos, aunque la víctima se asoció más al factor internalizante, mayormente con depresión, y el acosador al externalizante, ambos se relacionaron significativamente con conductas agresivas.

Puede considerarse entre las limitaciones del presente estudio que cuenta con una muestra limitada a un solo instituto y una sola fuente de información, el autoinforme de los adolescentes, cuando la mirada del docente podría ofrecer información importante respecto a estas situaciones. Por otra parte, futuros estudios podrían explorar las relaciones de mediación y moderación entre las variables aquí estudiadas.

Estudios como este van encaminados a esclarecer las complejas relaciones entre la personalidad, la psicopatología y las conductas de acoso escolar, lo que puede contribuir a abordajes preventivos y terapéuticos en relación con esta problemática. El bullying, además, contribuye a un mayor riesgo de psicopatología futura en los adolescentes (Hwang, Kim, Koh, Bishop y Leventhal 2017). Se ha sugerido que la efectiva prevención e intervención en casos de bullying deben tener en cuenta la complejidad de esta experiencia. Así, deben abordarse tanto las características individuales como los factores protectores y de riesgo y el contexto singular donde este fenómeno ocurre para poder promover relaciones sociales más saludables (Swearer y Hymel 2015). Los hallazgos en relación con el acoso escolar y sus consecuencias en la psicopatología general justifican la intervención temprana desde programas de prevención para estudiantes, además de políticas específicas contra el acoso escolar en las escuelas (Bang y Park 2017).

\section{Referencias bibliográficas}

Alonso, Cristina y Estrella Romero. 2017. «Aggressors and victims in bullying and cyberbullying: A study of personality profiles using the five-factor model». The Spanish journal of psychology, 76(20).

Bang, Young y Jae Hong Park. 2017. «Psychiatric disorders and suicide attempts among adolescents victimized by school bullying». Australasian psychiatry, 25(4): 376-380.

Díaz-Atienza, Francisco, Mercedes Prados Cuesta y Miguel Ruiz-Veguilla. 2004. «Relación entre las conductas de intimidación, depresión e ideación suicida en adolescentes: resultados preliminares». Revista de Psiquiatría y Psicología del Niño y del Adolescente, 4(1): 10-19.

Eastman, Meredith, Vangie Foshee, Susan Ennett, Daniela Sotres-Alvarez, Luz Reyes, Robert Faris y Kari North. 2018. «Profiles of internalizing and externalizing 
symptoms associated with bullying victimization». Journal of adolescence, 65 : 101-110.

Farmer, Thomas, Matthew Irvin, Luci Motoca, Man-Chi Leung, Bryan Hutchins, Debbie Brooks y Cristin Hall. 2015. «Externalizing and internalizing behavior problems, peer affiliations, and bullying involvement across the transition to middle school». Journal of Emotional and Behavioral Disorders, 23(1): 3-16.

Fernández-Pinto, Irene, Pablo Santamaría, Fernando Sánchez-Sánchez, Miguel Carrasco y Victoria del Barrio. 2015. SENA. Sistema de Evaluación de Niños y Adolescentes. Manual Técnico. Madrid: TEA Ediciones.

Goldbach, Jeremy, Paul Sterzing y Marla Stuart. 2018. "Challenging conventions of bullying thresholds: exploring differences between low and high levels of bullyonly, victim-only, and bully-victim roles». Journal of youth and adolescence, 47(3): 586-600.

Hwang, Soonjo, Young Kim, Yun-Joo Koh, Somer Bishop y Bennet Leventhal. 2017. «Discrepancy in perception of bullying experiences and later internalizing and externalizing behavior: A prospective study». Aggressive behavior, 43(5): 493502.

Kelly, Erin, Nicola Newton, Lexine Stapinski, Tim Slade, Emma Barrett, Patricia Conrod y Maree Teesson. 2015. «Suicidality, internalizing problems and externalizing problems among adolescent bullies, victims and bully-victims». Preventive medicine, 73: 100-105.

Klomek, Anat, Andre Sourander y Henrik Elonheimo. 2015. «Bullying by peers in childhood and effects on psychopathology, suicidality, and criminality in adulthood». The Lancet Psychiatry, 2(10): 930-941.

McCrae, Robert y Paul Costa. 2010. NEO Inventories for the NEO Personality Inventory-3 (NEO-PI-3), NEO Five-Factor Inventory-3 (NEO-FFI-3), NEO Personality Inventory-Revised (NEO-PI-R): Professional manual. Lutz, FL: Psychological Assessment Resources.

Mitsopoulou, Effrosyni y Theodoros Giovazolias. 2015. «Personality traits, empathy and bullying behavior: A meta-analytic approach». Aggression and violent behavior, 21: 61-72.

Nielsen, Morten y Stein Knardahl. 2015. «ls workplace bullying related to the personality traits of victims? A two-year prospective study». Work \& Stress, 29(2): 128-149.

Ortet, Generós, Patricia Escrivá, Manuel Ignacio Ibáñez, Jorge Moya, Helena Villa, Laura Mezquita, y María Ruipérez. 2010. «Versión corta de la adaptación española para adolescentes del NEO-PI-R (JS NEO-S)». International Journal of Clinical and Health Psychology: 10(2): 327-344.

Plopa, Mieczyslaw, Wojciech Plopa y Anna Skuzińska. 2017. «Bullying at work, personality and subjective well-being». Journal of Occupational Health Psychology, 22(1): 19-27.

Ramirez, Fuensanta Cerezo. 2015. «Agresores y Víctimas del Bullying. Desigualdades de género en la violencia entre escolares». Informació psicològica, (94): 48-59.

Ramírez, Luz, Alejandra Estefanía Ramírez-Pérez, Alexa Arellano y Andrea Trejo Vázquez. 2016. "Adolescencia (11-20 años): El uso de la violencia y el acoso en los escenarios escolares». PsicoEducativa: reflexiones y propuestas, 2(3): 77-84.

Sandoval Ato, Raul, Martin Vilela Estrada, Christian Mejía y José Caballero Alvarado. 2018. «Riesgo suicida asociado a bullying y depresión en escolares de secundaria». Revista chilena de pediatría, 89(2): 208-215.

Sekol, Ivana y David Farrington. 2016. «Personal characteristics of bullying victims in residential care for youth». Journal of aggression, conflict and peace research, 8(2): 99-113. 
Silberg, Judy, William Copeland, Julie Linker, Ashlee Moore, Roxann Roberson-Nay y Timothy York. 2016. «Psychiatric outcomes of bullying victimization: a study of discordant monozygotic twins». Psychological medicine, 46(9): 1875-1883.

Sterzing, Paul, Wendy Auslander, Allen Ratliff, Donald Gerke, Tonya Edmond y Melissa Jonson-Reid. 2017. «Exploring bullying perpetration and victimization among adolescent girls in the child welfare system: Bully-only, victim-only, bullyvictim, and noninvolved roles». Journal of interpersonal violence.

Swearer, Susan y Shelley Hymel. 2015. "Understanding the psychology of bullying: Moving toward a social-ecological diathesis-stress model». American Psychologist, 70(4): 344-353.

Tippett, Neil y Dieter Wolke. 2014. "Socioeconomic status and bullying: a metaanalysis». American journal of public health, 104(6): 48-59.

van Dijk, Anouk, Astrid Poorthuis y Tina Malti. 2017. "Psychological processes in young bullies versus bully-victims». Aggressive behavior, 43(5): 430-439.

van Geel, Mitch, Anouk Goemans, Fatih Toprak y Paul Vedder. 2017. «Which personality traits are related to traditional bullying and cyberbullying? A study with the Big Five, Dark Triad and sadism». Personality and Individual Differences, 106: 231-235.

Volk, Anthony, Katerina Schiralli, Xiaoyang Xia, Junru Zhao y Andrew Dane. 2018. «Adolescent bullying and personality: A cross-cultural approach». Personality and Individual Differences, 125: 126-132. 